provision for the education and instruction of children and young persons after the war, regard being had particularly to the interests of those (i) who have been abnormally employed during the war; (ii) who cannot immediately find advantageous employment; (iii) who require special training for employment. Among the twenty-three recommendations made by the committee are the following:-

(r) That a uniform elementary school leaving age of fourteen be established by statute for all districts, urban and rural and that all exemptions, total or partial, from compulsory attendance below that age be abolished.

(2) That steps be taken, by better stafing and other improvements in the upper classes of elementary sclools, to ensure the maximum benefit from the last years of school life.

(3) That it be an obligation on the local education authority in each area to provide suitable continuation classes for young persons between the ages of fourteen and eighteen, and to submit to the Board of Education a plan for the organisation of such a system, together with proposals for putting it into effect.

(4) That it be an obligation upon all young persons between fourteen and eighteen years of age to attend such day continuation classes as may be prescribed for them by the local education authority, during a number of hours to be fixed by statute, which should be not less than eight hours a week, for forty weeks in the year, with the exception of: $(a)$ Those who are under efficient full-time instruction in some other manner; (b) those who have completed a satisfactory course in a secondary school recognised as efficient by the Board of Education and are not less than sixteen; $(c)$ those who have passed the matriculation examination of a British university, or an equivalent examination, and are not less than sixteen; $(d)$ those who are under part-time instruction of a kind rot regarded as unsuitable by the Board of Education and entailing a substantially greater amount of $s^{r} u d y$ in the daytime than the amount to be required by statute.

(5) That all classes at which attendance is compulsory be held between the hours of $8 \mathrm{a} . \mathrm{m}$. and 7 p.m.

(6) That it be an obligation on all employers of roung persons under eighteen to give them the necessary facilities for attendance at the statutory continuation classes prescribed for them by the local education authority.

(i) That where there is already a statutory limitation upon the hours of labour, the permitted hours of labour be reduced by the number of those required for the continuation classes

(8) That the curriculum of the continuation classes include general, practical, and technical instruction, and that provision be made for continuous physical training and for medical inspection, and for clinical treatment where necessary, up to the age of eighteen.

(9) That suitable courses of training be established and adequate salaries be provided for teachers of continuation classes.

(Io) That the system of continuation classes come normally into operation on an appointed day as early as possible after the end of the war, and that the Board of Education have power to make deferring orders fixing later appointed days within a limited period, where necessary, for the whole or part of the area of any local education authority.

(II) That the State grants in aid of present as well as future expenditure on education be simplified and very substantially increased.

\section{RECENT PROGRESS IN SPECTROSCOPY."}

TEN years ago the subject of Prof. Crew's vicepresidential address was "Facts and Theories in Spectroscopy." Since that time some notable discoveries have been made and some remarkable theories have challenged attention. It is my purpose to review a few of the more important experimental results and to discuss the relations of some of them to theories brought before you in two recent vice-presidential addresses on "Atomic Theories of Radiation" and "The Theory of the Nucleus Atom." Inasmuch as it will be necessary to refer to them, I will restate the salient features of the theories which have attracted the most attention.

Planck derived an expression for the spectral energy distribution of black-body radiation from the assumption that the radiation was emitted and absorbed by electric oscillators in definite quanta, each equal to the frequency of the oscillator multiplied by a universal constant, $h$, the wirkungsquantum. Later he modified this theory so far as absorption is concerned. Einstein and others went farther in assuming that these quanta preserve their identity in their propagation through space, thus reviving a form of corpuscular theory. This extreme view has been generally abandoned, but it has been found impossible to explain away the wirkungsquantum $h$. It appears in too many relations to be the result of chance. The work of Millikan in particular proves the exact validity of Einstein's relation $V e=h\left(v-v_{0}\right)$ in the photoelectric effect, in which $V e$ is the measure of the emission energy of the electrons, $v$ the frequency of the incident light, and $v_{0}$ the minimum frequency which will cause emission of electrons. A similar relation appears to hold good in many cases of X-ray and light spectra. It seems probable that this constant depends upon atomic structure only, and affects radiation through space only in so far as emission and absorption are determined by atomic structure.

The theory of the nucleus atom is likewise of fundamental importance in spectroscopy. The worls of Rutherford and others leaves no escape from the conclusion that the nucleus of the atom is a concentrated group of positive charges and electrons, with an excess of positive elementary charges approximately equal to half the atomic weight, while the same number of electrons circulate about the nucleus in rings. The spectroscopist must try to fit his theories to these probable facts, but he is met at the outset with apparently insuperable difficulties in accounting for the stability of such atoms and for the manifold complexity of spectra according to accepted electrodynamical laws. Bohr cut the Gordian knot by supposing that the classic laws apply only to conditions of stability, when no energy is radiated, and that radiation attends the transition of an electron from one state of stability to another, the frequency being determined by the relation that $h$ multiplied by the frequency is equal to the difference between the energies of the system in the two stable states. In the case of hydrogen, to which he assigns one radiating electron and one nucleus charge, it is difficult to account for the existence of so many stable states, for the failure to radiate while subject to uniform radial acceleration, and for monochromatic radiation while passing between two positions of stability. Nevertheless, Bohr derived an expression like that of Rydberg which locates accurately not only the Balmer series, but also an infra-red and an ultraviolet series predicted by Ritz and found by Paschen

1 Address delivered to Section B-Physics-of the American Association for the Advancement of Science at the New York meeting, December, rgr6 by the chairman of the Section, Prof. E. P. Lewis. 
and by Lyman, respectively. His attempt to apply the same method to helium led to results which are still in dispute, and will be referred to later.

In reviewing recent progress we may begin with that field in which the United States has taken a leading part-that of astrophysics. This domain belongs as much to the physicist as to the astronomer. The heavenly bodies are laboratories on a vast scale, in which nature has provided conditions of temperature, pressure and electrical state which we may never hope to rival on the earth. The spectroscope gives us data from which it may be possible to form some idea of these conditions by comparison with our feeble laboratory imitations of celestial phenomena, and conversely, the latter may aid in the interpretation of terrestrial phenomena.

One of the most fruitful astronomical applications of the spectroscope is to the determination of velocities in the line of sight, by the Doppler-Fizeau principle. A large mass of such data has been collected, from which some important generalisations have been derived. For example, Campbell has determined the velocity and direction of motion of the solar system through space, and has found a remarkable and as yet unexplained relation between the velocities of stars and their apparent age, the redder and presumably older stars and a class of nebulæ having in general the greater velocities. It likewise appears that two immense star streams are crossing each other in the Milky Way. Many spectroscopic binaries have been discovered and their orbits determined, and recently there have been found remarkable displacements and rotations in nebulæ which may throw some light on the nature and destiny of these bodies. The spectroscope has enabled astronomers to undertake the ambitious task of tracing the course of stellar evolution.

The most ingenious and fruitful device for studying the sun is the spectroheliograph, invented by Hale in r892. With this instrument photographs of the distribution of a given constituent of the solar atmosphere may be obtained by restricting the light falling on the photographic plate to the wave-length of one of the characteristic lines of the element. The configuration of the hydrogen clouds in the neighbourhood of sunspots led Hale to suspect vortical motions in such regions. In 1908 the study of a number of plates, which showed that hydrogen flocculi were actually drawn into these spots from great distances, proved without question that sunspots are cyclonic areas of enormous extent. 'Thus the long-disputed question as to the nature of sunspots was answered, but this was not all. Vapours which emit or absorb line spectra are ionised, and as the more mobile electrons would diffuse more rapidly to higher levels than the positive ions, Hale inferred that the immense whirls of electrified vapours in the neighbourhood of the spots must cause a radial magnetic field. If such fields are sufficiently intense, the longitudinal Zeeman effect should be produced. As a matter of fact, the spectrum of light from the spots is characteristically different from that of the surrounding photosphere, one of these peculiarities being the doubling of many lines. As Hale anticipated, an examination of the state of polarisation of such lines showed them to be circularly polarised, and the direction indicated that the whirling vapour was negatively electrified. Hale likewise sought for the more minute effects which might be expected from the rotation of the solar atmosphere as a whole. A study of the breadth of spectral lines at different latitudes and the detection of traces of circular polarisation at their edges showed that the sun possesses a magnetic field with polarity corresponding to that of the earth, but of much greater intensity. Aithough the atmospheric conditions on the earth are very different from those on the sun, it is possible that these investigations may assist us in solving the baffling problem of the earth's magnetism.

One of the most impressive facts revealed by the spectroscope is the substantial identity of constitution of the heavenly bodies. Everywhere we find evidence of the existence of such elements as hydrogen, sodium, calcium, and iron. But we also find an infinitude of differences in the appearance of the lines, which we must attribute to differences of temperature, vapour density, pressure and electrical condition. It is suggestive to find that the spectrum of some stars resembles that of the arc, of others that of the spark. We may hope by comparing the spectra of these bodies with those produced in our laboratories under varied conditions to reach some conclusions regarding their physical state. The Mount Wilson physical laboratory is doing much valuable work of this kind.

In the spectra of the solar corona and of nebulæ and nebulous stars certain lines are found which do not belong to known elements. This need not indicate any fundamental differences between the lifehistory of such bodies and that of the older stars. Twenty-five years ago Lockyer's views regarding the dissociation of elements in the stars were treated with levity by most physicists and astronomers. To-day such notions are held to be quite rational. The more elementary forms of matter would naturally be of small atomic weight, and hence would diffuse to higher levels than the heavier elements, and might ultimately escape into space. If it were not for the fact that it is held captive in chemical combinations, we should know nothing of hydrogen. Helium first revealed itself to us through its solar lines, and would still be otherwise unknown to us were it not for its continuous production in radioactive processes. The elements giving the spectra of the corona and of the nebulæ are presumably of small atomic weight, and are possibly the units out of which more complex known elements are built, in later stages of development; or they may be, conversely, the results of the disintegration of such elements. It is not impossible that in the future we may detect traces of these elements on the earth or manufacture them by some powerful disintegrative process. Meanwhile, deductions from known relations between frequencies of the spectral lines, their breadth, and the atomic weight of the elements may give us some clue to their atomic weights. Nicholson has succeeded in constructing hypothetical atoms with given nuclear charges and electron ring systems which give with remarkable accuracy the positions of the lines of the cornna and nebulæ. Rayleigh showed from kinetic theory and Michelson proved experimentally that at low pressures the width of lines may be entirely due to Doppler displacements, which vary directly as the square root of the absolute temperature and inversely as the square root of the atomic weight. Buisson and Fabry have verified this law and applied it to the study of nebulæ. The width of certain lines, determined from the limit of interference, indicates that the temperature of the Orion nebula is about 15,000 degrees, and that two groups of lines are due to atoms of weights 2.72 and between $\mathrm{r}$ and 2 respectively. This is a remarkable confirmation of Nicholson's previous conclusion that the emission centres are of atomic weights 2.95 and $\mathrm{x} \cdot 3 \mathrm{I}$.

During the past ten years the boundaries of the known spectrum have been greatly extended in both directions. The difficulties of investigation in the infra-red are very great, but by the methods of reststrahlen and of focal isolation Rubens, worlsing in succession with Nichols, Wood and von Baeyer, has isolated and measured certain regions of great wave-length. The longest wave-length measured is NO. 2475, VOL. 99] 
about $0.3 \mathrm{~mm}$., while the shortest Hertzian waves so far obtained are $2 \mathrm{~mm}$. long. The study of line radiation in this region is even more difficult, but Paschen and his pupil, the American Randall, have succeeded in measuring many lines extending to about 90,000 Alngström units.

In the ultra-violet Lyman has extended the region first made known to us by Schumann to a wavelength of about 600 Angström units. Beyond this point it is difficult to go, on account of absorption, lack of sensitiveness of the photographic plate, and small reflecting power of speculum metal. Gratings ruled on silicon and photoelectric detectors may enable us to bridge the gap between these waves and the much shorter ones which may be examined with the aid of nature's diffraction gratings, crystals which have made the study of X-ray spectra possible.

Of all the discoveries of recent years, that of the wave nature of the X-rays and of a practical method of examining their spectra is the most remarkable and the most important, for it has revealed to us the most fundamental radiations of the elements and has viven us a glimpse into the very heart of the atom. In quick succession Laue and his pupils demonstrated the diffraction effects produced by crystals, the Braggs showed how reflection might be employed to isolate waves of different lengths by a principle similar to that producing colours of thin plates, but of far greater resolving power by reason of the greater number of effective reflecting surfaces, and Moseley photographed many characteristic spectra by an extraordinarily simple method. He found that the principal lines in the spectra of a large number of elements were connected by a remarkably simple relation, namely that the square roots of the frequencies are proportional to the ordinal numbers, which increase by one in passing from one number of a periodic group to the next. When there are anomalies between the atomic weight and the place of an element in a group, this anomaly disappears when the atomic number rather than the atomic weight is considered. This work has been extended by others, notably by Siegbahn and Friman, to include nearly all the known elements between sodium and uranium, inclusive, with the result that all the atomic numbers between hydrogen and uranium are accounted for, with the exception of six gaps. As interpreted by Bohr's theory, the ordinal number which determines the frequency is the excess number of positive elementary charges in the nucleus, and these results are, therefore, in complete harmony with the theory of the nuclear atom developed by Rutherford, van den Broek, Soddy, and others. The comparison of the X-ray spectrum of lead obtained by Siegbahn with the gamma-ray spectrum of radium $B$ obtained by Rutherford and Andrade shows the identity of ten of the principal lines. This strikingly confirms the accepted theory of isotopes, or elements of different atomic weights, which are chemically and spectroscopically alike beause they have the same resultant nuclear charge.

The positions of the principal lines are consistent with Bohr's general formula, but perhaps this relationship is purely formal. But whether or not this theory applies, apparently we cannot dispense with the wirkungsquantum. In addition to the characteristic X-radiation of an element, there is a continuous spectrum, with a sharply defined boundary on the side of shorter wave-lengths. The investigations of Duane, Hull and D. L. Webster have shown that this boundary is accurately defined by Einstein's relation $V e=h v$ for fields up to I I0,000 volts. Such a simple law does not hold for the characteristic radiations; but Webster has shown that they do not appear until the voltage somewhat exceeds that demanded by the EinNo. 2475, VOL. 99] stein relation. The longest $\mathrm{X}$-waves so far discovered by Siegbahn are about 12 Angström units in length, so that there is not a very great gap between them and the shortest waves discovered by Lyman. The investigation of this region is difficult, but undoubtedly means will be found to attain success. Much also remains to be done in the study of details of X-ray spectra, which contain many weak lines, and possibly bands, which have not so far been carefully examined.

During the past ten years great advance has been made in our knowledge of spectral series. Rydberg, Ritz, Paschen, Fowler and others have shown that a generalised form of the Balmer equation, with Rydberg's universal constant and a few special constants, is capable of wide application. Different combinations of a few constants have been found to give a number of related series, and many new lines so predicted have been found. The common limit and other numerical relationships between different series of the same element indicates that the different emission centres have some dynamic coupling and Rydberg's universal constant indicates a structural element common to all substances. According to Bohr, this quantity is a function of the electronic and atomic mass, the elementary electrical charge, and the wirkungsquantum $h$, and should slightly increase with increasing atomic weight. As it is commonly assumed that it is an absolute constant, careful measurements may furnish a test of the validity of Bohr's theory.

The relationships of frequency to atomic number found by Moseley recalls that Ramage, Watts, Runge and Precht and Hicks have found linear relationships between the squares of the atomic weights and the frequencies or frequency differences of homologous lines in the spectra of elements of the same group. Ives and Stuhlmann have shown that in some cases the results are improved by substituting atomic numbers for atomic weights, but the relationship is evidently not so simple as in the case of X-ray spectra.

The discovery of the Zeeman effect and the explanation of its simpler forms by Lorentz was the first step toward a rational spectroscopic theory. The later discovered complexities and anomalies, while they may defy mathematical analysis, do not lessen our confidence in the theory, for they are what we might expect as a result of complicated atomic structure. The same intellectual satisfaction does not attend the discovery of the analogous effect of an clectric field, because the simplest cases are so complex that they cannot be adequately explained by any theory yet proposed. The possibility of such an effect had long been the subject of speculation, but Stark was the first to realise and attain the necessary conditions for its occurrence. Lo Surdo also discovered it in the neighbourhood of the cathode in capillary tubes. As in the case of the Zeeman effect, the phenomena are different when viewed transversely and parallel to the field. In each case the lines are split into a number of components, the number being different for different lines, even for those belonging to the same series. In the transverse effect the components are planepolarised in hydrogen and helium, the stronger central lines vibrating at right angles to the field, and the stronger outer components vibrating parallel to the field. A remarkable relation is found for the series lines of hydrogen, helium and lithium. For each the number of principal normal components appears to be equal to the ordinal number of the line in the series. Higher dispersion shows that in the case of hydrogen each component is double. If this rule holds good throughout the series, the last known line, the twentyeighth, would have fifty-six such components, an equal number polarised at right angles to these, and a 
number of weaker components of both kinds-truly a formidably complicated system. In general, the longitudinal components appear to be unpolarised, although Miss Howell has found some anomalies with lithium and calcium. In some cases the components are unsymmetrical both in position and in intensity. Of all the other elements investigated, mercury alone shows a slight broadening. It might be expected that the great nuclear charges of heavy atoms would diminish the effect of an external field. The inverse absorption effect has so far not been observed.

Long before the Stark effect was observed Voigt showed that such results might be expected from quasielastic forces in the atom and the stresses produced by the field. Schwarzschild has attempted to explain it by the ordinary laws of electrodynamics, and Warburg, Gehrcke, Garbasso and Bohr by Bohr's theory. Each attempt was successful in some respects, but each failed to account fully for all the components, their displacements and their state of polarisation, and all the theories assign the same number of components to each line of a series, whereas one of the most significant features is the progressive difference in number of components, displacements and relative intensities in passing from one line to another. Stark not only rejects them all, but is led by his study of the phenomenon to abandon finally the quantum and light-cell theories, because he considers that he has proved that the greatest possible energy which an electron can acquire in its orbit falls far short of one energy quantum. Moreover, he argues that it seems impossible to explain the phenomenon in terms of Bohr's one electron. He concludes that a number of electrons must take part in the emission of a single line, each having the same frequency under ordinary conditions or in a magnetic field, but different frequencies when displaced unsymmetrically in an electric field. It is difficult, however, to understand why hydrogen has only one detachable electron if Stark's view is correct.

It has already been mentioned that at lorv pressures the width of lines may be ascribed entirely to the Doppler effect. The great broadening at higher pressures has never been explained, but it has been assumed that damping, collisions and rotations all play a part. Stark suggests that it may be largely due to atomic electric fields, which may exercise a large influence when the atoms are crowded together. It seems significant that the broadening increases with the ordinal number of a line in a series, is often unsymmetrical, and diminishes with increasing atomic weight in most cases, quite in harmony with the effects of an electric field. Nicholson and Merton have found that the broadening of hydrogen lines is in quantitative agreement with Stark's suggestion.

With changes in vapour density, pressure, temperature or the mode of excitation lines belonging to one series may weaken or disappear, other lines may be strengthened, and new lines may appear. We must assume that different groups of lines are due to different emission centres. These differences must depend upon the size of the particles, or upon the number and arrangement of electrons. Any theory must take account of the molecular or atomic state or the electrical charge of the emission centres. In some cases we have rather definite information on these points.

A number of elements emit band spectra under some conditions, line spectra under others. One conclusion which seems to be well established is that band spectra are emitted by molecules, line spectra by atoms. Universally, we find that compounds give band spectra, never line spectra. If a compound is dissociated by the discharge the line spectrum of one or both constituents appears. Elements give band spectra with feeble excitation, line spectra when the discharge is No. 2475, VOL. 99] so intense as to cause dissociation. It seems reasonable to infer that the band spectra of elements is likewise associated with the molecular condition. In the case of monatomic elements which give both band and line spectra electrical conditions must determine the nature of the radiation.

(To be continued.)

\section{CNIVERSITY AND EDUCATIONAL INTELLIGENCE.}

The war has brought women students into prominence in Germany. They form a third of the actual number of students in residence at the twentytwo universities of the Empire, and one-tenth of the total number of registered students. During the winter of $19 \mathrm{I} 6-\mathrm{I} 7$ there were more women than men at several German universities, e.g. Marburg and Münster; in Bonn, Frankfurt, Munich, Heidelberg, and Jena the women formed half the students, while they were in a minority at Strassburg, Leipzig, Breslau, and Giessen. Altogether, there were 5757 women undergraduates at the German universities during the last term, distributed as follows :Literature and history, 2789; mathematics and science, 1036 ; medicine, 1479; dentistry, 64; economics and agriculture, 225; law, 116; Protestant theology, I8; and pharmacy, 30 .

THE committee appointed to consider arrangements for post-graduate teaching in the Calcutta University has, we learn from the Pioneer Mail, presented a report dealing exhaustively with that subject. In summing up the recommendations the committee states that the proposals, in the main, amount to the acceptance of two fundamental principles: $(a)$ an intimate association and co-operation between the college and the university staffs is imperative in the interest of all concerned and of the development of higher teaching; $(b)$ it is necessary to oonstitute a suitable organisation within which the teachers will be enabled by discussion among themselves efficiently to conduct the teaching and examination of graduates. Beyond this, says the report, the committee has been unable to go and has refrained from commenting on the wider problems which confront the University.

\section{SOCIETIES AND ACADEMIES.}

\section{LONDON.}

Royal Society. March 22.-Sir J. J. Thomson, president, in the chair.-J. C. Mottram and Dr. S. Russ : Observations and experiments on the susceptibility and immunity of rats towards Jensen's rat sarcoma. Observations have been made upon the modes of growth of Jensen's rat sarcoma following inoculation. There is a gradual transition from those cases in which the tumours spontaneously disappear to those in which they grow in a uniformly progressive manner. The experimental production of the immune condition can be brought about in several ways. Animals made refractory to the growth of the tumour have been given various doses of X-rays; the effect of such irradiation upon the blood was to cause a marked reduction in the number of lymphocytes. Over suitable conditions of exposure it has been possible to destroy the immune condition and thus convert refractory into tumour-bearing animals. There is a tendency for the immune condition to be restored. Histological and other evidence is brought forward which indicates that the failure of sarcoma cells to grow in an immune animal is due to an active resistance thereto on the part of the host.-S. Pickering: Problems bearing on rcsidual affinity. It has been ascertained that the 